\title{
Krausz dimension and its generalizations in special graph classes
}

\author{
Olga Glebova \\ Yury Metelsky \\ Pavel Skumst
}

Department of Mechanics and Mathematics, Belarus State University, Minsk, Republic of Belarus.

received 12 th April 2011, revised 25 th June 2012, accepted 25 th February 2013.

A $\operatorname{Krausz}(k, m)$-partition of a graph $G$ is a decomposition of $G$ into cliques, such that any vertex belongs to at most $k$ cliques and any two cliques have at most $m$ vertices in common. The $m$-Krausz dimension $k d i m_{m}(G)$ of the graph $G$ is the minimum number $k$ such that $G$ has a $\operatorname{Krausz}(k, m)$-partition. In particular, 1-Krausz dimension or simply Krausz dimension $\operatorname{kdim}(G)$ is a well-known graph-theoretical parameter.

In this paper we prove that the problem " $k \operatorname{dim}(G) \leq 3$ " is polynomially solvable for chordal graphs, thus partially solving the open problem of P. Hlineny and J. Kratochvil. We solve another open problem of P. Hlineny and J. Kratochvil by proving that the problem of finding Krausz dimension is NP-hard for split graphs and complements of bipartite graphs. We show that the problem of finding $m$-Krausz dimension is NP-hard for every $m \geq 1$, but the problem " $k \operatorname{dim}_{m}(G) \leq k$ " is is fixed-parameter tractable when parameterized by $k$ and $m$ for $(\infty, 1)$-polar graphs. Moreover, the class of $(\infty, 1)$-polar graphs with $\operatorname{kdim}_{m}(G) \leq k$ is characterized by a finite list of forbidden induced subgraphs for every $k, m \geq 1$.

Keywords: Krausz dimension, intersection graph, linear k-uniform hypergraph, chordal graph, split graph, polar graph

\section{Introduction}

In this paper we consider finite undirected graphs without loops and multiple edges. The vertex and the edge sets of a graph (hypergraph) $G$ are denoted by $V(G)$ and $E(G)$ respectively. $N(v)=N_{G}(v)$ is the neighborhood of a vertex $v$ in $G$ and $\operatorname{deg}(v)$ is the degree of $v$. Let $G(X)$ denote the subgraph of $G$ induced by a set $X \subseteq V(G)$ and $\operatorname{ecc}_{G}(v)$ is the eccentricity of a vertex $v \in V(G)$.

A Krausz partition of a graph $G$ is a decomposition of $G$ into cliques, which are called clusters of the partition, such that every edge of $G$ belongs to exactly one cluster. If every vertex of $G$ belongs to at most $k$ clusters, then the partition is called Krausz k-partition. The Krausz dimension $k \operatorname{dim}(G)$ of the $\operatorname{graph} G$ is a minimal $k$ such that $G$ has Krausz $k$-partition.

Krausz $k$-partitions are closely connected with the representation of a graph as an intersection graph of a hypergraph. The intersection graph $L(H)$ of a hypergraph $H=(V(H), E(H))$ is defined as follows:

\footnotetext{
${ }^{\dagger}$ Email: skumspegmail.com

1365-8050 (c) 2013 Discrete Mathematics and Theoretical Computer Science (DMTCS), Nancy, France
} 
1. the vertices of $L(H)$ are in a bijective correspondence with the edges of $H$;

2. two vertices are adjacent in $L(H)$ if and only if the corresponding edges have a nonempty intersection.

Hypergraph $H$ is called linear if any two of its edges have at most one common vertex; $k$-uniform if every edge contains $k$ vertices.

The multiplicity of the pair of vertices $u, v$ of the hypergraph $H$ is the number $m(u, v)=\mid\{\mathcal{E} \in E(H)$ : $u, v \in \mathcal{E}\} \mid$; the multiplicity $m(H)$ of the hypergraph $H$ is the maximum multiplicity of the pairs of its vertices. So, linear hypergraphs are the hypergraphs with the multiplicity 1.

Denote by $H^{*}$ the dual hypergraph of $H$ and by $H_{[2]}$ the 2-section of $H$, i.e., the simple graph obtained from $H$ by the transformation of each edge into a clique. It follows immediately from the definition that

$$
L(H)=\left(H^{*}\right)_{[2]} .
$$

First this relation was implicitly formulated by C. Berge in [3]. (1) implies that a graph $G$ has Krausz $k$-partition if and only if it is an intersection graph of linear $k$-uniform hypergraph.

A graph $G$ is called polar [7, 21] if there exists a partition of its vertex set

$$
V(G)=A \cup B, A \cap B=\emptyset
$$

such that all connected components of the graphs $\bar{G}(A)$ and $G(B)$ are complete graphs. The partition $(A, B)$ is called bipartition. If, in addition, $\alpha$ and $\beta$ are fixed integers and the orders of connected components of the graphs $\bar{G}(A)$ and $G(B)$ are at most $\alpha$ and $\beta$ respectively, then the polar graph $G$ with bipartition (2) is called $(\alpha, \beta)$-polar [5]. Given a polar graph $G$ with bipartition [2], if the order of connected components of the graph $\bar{G}(A)$ (the graph $G(B)$ ) is not restricted above, then the parameter $\alpha$ (respectively $\beta$ ) is supposed to be equal $\infty$. Thus an arbitrary polar graph is $(\infty, \infty)$-polar, and $(1,1)$ polar graphs are exactly well-known split graphs.

Denote by $K D I M(k)$ the problem of determining whether $k \operatorname{dim}(G) \leq k$ and by $K D I M$ the problem of finding the Krausz dimension.

Graphs with Krausz dimension at most 2 are exactly line graphs. The class of line graphs has been studied for a long time. It is characterized by a finite list of forbidden induced subgraphs [1], efficient algorithms for solving the problem $K D I M(2)$ and constructing the corresponding Krausz 2-partition are also known [6, 12, 18, 19].

The situation changes radically if one takes $k=3$ instead of $k=2$ : the problem $\operatorname{KDIM}(k)$ is NP-complete for every fixed $k \geq 3$ [9]. The case $k=3$ was studied in the different papers [10, 15, 16, 17, 20], and several graph classes, where the problem $K D I M(3)$ is polynomially solvable or remains NP-complete, were found.

In [9] P. Hlineny and J. Kratochvil studied the computational complexity of the Krausz dimension in detail. Besides other results, the following findings were obtained in their paper:

R1) The problem $K D I M$ is polynomially solvable for graphs with bounded treewidth. In particular, it is polynomially solvable for chordal graphs with bounded clique size.

R2) For the whole class of chordal graphs, the problem $K D I M(k)$ is NP-complete for every $k \geq 6$.

R3) For every fixed $k$ the problem $K D I M(k)$ is polynomially solvable in the class of split graphs. Note, that this result was independently obtained in [13]. 
Note, that the study of Krausz dimension of split graphs were motivated by results of L. Beineke and I. Broere [2], who studied the Krausz dimension of complete graph without one edge, which appeared to be non-trivial problem.

Based on their results, P. Hlineny and J. Kratochvil posed the following open problems:

P1) decide the complexity of $K D I M(k)$ restricted to chordal graphs for $k=3,4,5$;

P2) decide the complexity of $K D I M$ for split graphs and complements of bipartite graphs.

In this paper we solve Problem P2 2 and partially solve Problem P1 In Section 2 we prove that the problem $\operatorname{KDIM}(3)$ is polynomially solvable in the class of chordal graphs. In Section 3 we show that the problem $K D I M$ is NP-hard for both classes of split graphs and complements of bipartite graphs.

In Section 4 we consider a natural generalization of the Krausz dimension. A Krausz $(k, m)$-partition of a graph $G$ is a decomposition of $G$ into cliques, which are called clusters of the partition, such that any vertex belongs to at most $k$ clusters, and any two clusters have at most $m$ vertices in common. As shown above, the relation (1) implies the following statement:

Proposition 1 A graph $G$ has Krausz $(k, m)$-partition if and only if it is the intersection graph of a $k$-uniform hypergraph with the multiplicity at most $m$.

The $m$-Krausz dimension $k \operatorname{dim}_{m}(G)$ of the graph $G$ is the minimum $k$ such that $G$ has a Krausz $(k, m)$ partition. The Krausz dimension in those terms is the 1-Krausz dimension.

Denote by $K D I M_{m}$ the problem of determining the $m$-Krausz dimension of graph, by $K D I M_{m}(k)$ the problem of determining whether $k \operatorname{dim}_{m}(G) \leq k$ and by $L_{k}^{m}$ the class of graphs with a $\operatorname{Krausz}(k, m)$ partition. It was proved in [11] that the class $L_{3}^{m}$ could not be characterized by a finite set of forbidden induced subgraphs for every $m \geq 2$, but the complexity of the problem $K D I M_{m}$ for an arbitrary $m$ has not been established yet. We prove that the problem $K D I M_{m}$ is NP-hard for every $m \geq 1$.

The class $L_{k}^{m}$ is hereditary, i.e., closed with respect to deleting the vertices. Therefore it can be characterized in terms of forbidden induced subgraphs. We prove that for every fixed integers $m, k$ such finite characterization of the class exists when restricted to $(\infty, 1)$-polar graphs. In particular, it follows that the problem $K D I M_{m}(k)$ is fixed-parameter tractable when parameterized by $k$ and $m$. It is the strong generalization of the result $R 3$. Note also, that the class of $(\infty, 1)$-polar graphs could be recognized in polynomial time [5].

\section{Krausz 3-partitions of chordal graphs}

Let $F$ be a family of cliques of graph $G$. The cliques from $F$ are called clusters of $F$. Denote by $l_{F}(v)$ the number of clusters from $F$ covering the vertex $v$ and by $C\left(v_{1}, \ldots, v_{r}\right)$ the cluster containing vertices $v_{1}, \ldots, v_{r}$.

A maximal clique with at least $k^{2}-k+2$ vertices is called a $k$-large clique. For such cliques the following statement holds:

Lemma $2([9,10,17 \mid)$ Any $k$-large clique of a graph $G$ belongs to every Krausz $k$-partition of $G$.

Further in this section 3-large clique will be called simply large clique.

Let $G$ be a graph with $k \operatorname{dim}(G) \leq 3$ and $Q$ be some its Krausz 3-partition. Any subset $F \subseteq Q$ is called a fragment of the Krausz 3-partition $Q$ or simply a fragment. 
Let $F$ be some fragment of Krausz 3-partition $Q$ and $H$ be the subgraph of $G$ obtained by deleting edges covered by $F$. Further we will use the notation $H=G-E(F)$. The set $F$ could be empty. Fix some vertex $a \in V(H)$ and positive integer $k$. Denote by $B_{k}[a]$ the $k$ th neighborhood of $a$ in $H$, i.e. the set of vertices at distance at most $k$ from $a$. In particular, $N(a)=B_{1}[a] \backslash\{a\}$ denote the set of neighbors of $a$. A family of cliques $F_{k}(a)$ in $H$ is called $(a, k)$-local fragment or simply a local fragment, if

1. every edge with at least one end in $B_{k}[a]$ is covered by some cluster of $F_{k}(a)$,

2. every vertex $v \in B_{k}[a]$ belongs to at most $3-l_{F}(v)$ clusters of $F_{k}(a)$,

3. every two clusters of $F_{k}(a)$ have at most one common vertex.

A clique $C$ of $H$ is called special if $C$ is a cluster of every $(a, k)$-local fragment for some $a$ and $k$. In particular, by Lemma 2 large cliques are special.

The following statement is evident.

Lemma 3 Let $G$ be a graph with a Krausz 3-partition $Q, F$ be a fragment of $Q$ and $H=G-E(F)$. Then the following propositions hold:

1. If $\operatorname{deg}(v) \geq 19$ for some vertex $v \in V(G)$, then $v$ is contained in some large clique.

2. If $l_{F}(v)=2$, then $C=N_{H}(v) \cup\{v\}$ is a special clique.

3. If $v \in B_{k}[a]$ is adjacent to at least $4-l_{F}(v)$ vertices of the cluster $C$ of some local fragment $F_{k}(a)$, then $v \in C$.

4. For every $a \in V(H)$ and every $k$ there exists at least one $(a, k)$-local fragment;

5) If the clique $C$ is special, then $F \cup\{C\}$ is a fragment.

Proof:

1. If $v$ is contained in clusters $C_{1}, C_{2}, C_{3} \in Q$, then $\left|C_{1}\right|+\left|C_{2}\right|+\left|C_{3}\right|=\operatorname{deg}(v)+3 \geq 22$, which implies, that at least one of the clusters $C_{1}, C_{2}, C_{3}$ has more than 7 vertices.

2. If $F_{k}(a)$ is a local fragment, then by the definition $v$ belongs to at most one cluster $C \in F_{k}(a)$. Therefore all neighbors of $v$ in $H$ belong to $C$, i.e. $C=N_{H}(v) \cup\{v\}$.

3. If $v \in B_{k}[a]$ is adjacent to vertices $v_{1}, \ldots, v_{4-l_{F}(v)} \in C \in F_{k}(a)$, but $v \notin C$, then the edges $v v_{1}, \ldots, v v_{4-l_{F}(v)}$ should be covered by different clusters of $F_{k}(a)$. It contradicts (2).

4. The family of cliques $X=\left\{C \in Q \backslash F: C \cap B_{k}[a] \neq \emptyset\right\}$ is a local fragment.

5. Let $X$ is the same as in the proof of 4). Since $C$ is special, $C \in X$ and therefore $C \in Q \backslash F$.

Lemma 4 Let $G$ be a chordal graph and $S$ be its cycle with length at least 4 . Then for every two consecutive vertices of $S$ at least one of them belongs to some chord. 
Proof: Let $S=\left(a_{1}, \ldots, a_{k}\right), a_{i} a_{i+1} \in E(G)$, indices are taken modulo $k$. The statement of lemma is obvious, if $k=4$. Let $k \geq 5$. Without lost of generality consider two adjacent vertices $a_{k}, a_{1}$ of $S$. If the statement of lemma is not true, then one can choose the chord $a_{p} a_{q}, 1<p<q<k$ such, that $(p-1)+(k-q)$ is minimal. But then $\left(a_{1}, \ldots, a_{p}, a_{q}, \ldots, a_{k}\right)$ is a chordless cycle.

Denote by $l c(H)$ the length of a longest induced cycle of the graph $H$.

Lemma 5 Let $G$ be a chordal graph with a Krausz 3-partition $Q, F$ be a fragment of $Q$ and $H=$ $G-E(F)$. Let further there be no (a,2)-local fragments, which contain special cliques in $H$. Then $l c(H) \leq 6$.

Proof: Suppose the contrary, i.e., let $a_{1}, \ldots, a_{k}$ form the induced cycle $S \cong C_{k}$ in $H, k \geq 7, a_{i} a_{i+1} \in$ $E(H)$, indices are taken modulo $k$.

Since each $a_{i}$ has two nonadjacent neighbors in $H$, in every local fragment with center in $a_{i}$ it is covered by at least 2 clusters. It implies $l_{F}\left(a_{i}\right) \leq 1$ for every $i=1, \ldots, k$.

As $G$ is a chordal graph, there exist chords of the cycle $S$ covered by the fragment $F$. As $l_{F}\left(a_{i}\right) \leq 1$, for every vertex $a_{i}$ chords incident to this vertex are covered by exactly one cluster of $F$. It implies that no pair of chords has the form $\left\{a_{i} a_{j}, a_{i} a_{j+1}\right\}$, since in this case the vertices $a_{i}, a_{j}, a_{j+1}$ are covered by one cluster of $F$ and thus the edge $a_{j} a_{j+1}$ should be covered by $F$.

Assume without loss of generality that a chord of $S$ contains $a_{1}$. Let us show that all chords of $S$ are covered by the cluster $C_{\text {chord }} \supseteq\left\{a_{1}, a_{3}, \ldots, a_{k-1}\right\}$. Indeed, suppose that some chords of $S$ are covered by the cluster $C \supseteq\left\{a_{i_{1}}, \ldots, a_{i_{r}}\right\}, i_{1}<i_{2}<\ldots<i_{r}, i_{1}=1, C \neq C_{\text {chord }}$. Then there exists $1 \leq p<r$ such that $i_{p+1}-i_{p} \geq 3$. So, $\left(a_{i_{p}}, a_{i_{p}+1}, \ldots, a_{i_{p+1}-1}, a_{i_{p+1}}\right)$ is a cycle of length at least 4 , in which at least one of the vertices $a_{i_{p}}, a_{i_{p+1}}$, say $a_{i_{p}}$, belongs to some chord according to Lemma 4 . That chord should be covered by a cluster $C^{\prime} \in F, C^{\prime} \neq C$. So, we have $l_{F}\left(a_{i_{p}}\right) \geq 2$, the contradiction.

In particular, this proposition implies the following:

1. $k$ is even;

2. for any odd $i$ and even $j$ such that $a_{i} a_{j}$ is not the edge of $S$, the vertices $a_{i}$ and $a_{j}$ are nonadjacent in $G$. It follows from the fact, that otherwise $l_{F}\left(a_{i}\right) \geq 2$.

Since there are no special cliques in $H$ there exist two different local fragments $F_{2}\left(a_{1}\right) \supseteq\left\{C\left(a_{1}, a_{2}\right)\right.$, $\left.C\left(a_{1}, a_{k}\right)\right\}$ and $F_{2}^{\prime}\left(a_{1}\right) \supseteq\left\{C^{\prime}\left(a_{1}, a_{2}\right), C^{\prime}\left(a_{1}, a_{k}\right)\right\}$, such that without loss of generality $C\left(a_{1}, a_{2}\right) \backslash$ $C^{\prime}\left(a_{1}, a_{2}\right) \neq \emptyset$.

Let $v \in C\left(a_{1}, a_{2}\right) \backslash C^{\prime}\left(a_{1}, a_{2}\right)$. Then $v \in C^{\prime}\left(a_{1}, a_{k}\right)$ and therefore $a_{1} v, a_{2} v, a_{k} v \in E(H)$.

The vertices $a_{2}, v, a_{k}, a_{k-1}, a_{3}$ form a cycle in $G$. It should have at least 2 chords. Since $a_{2} a_{k-1}, a_{3} a_{k} \notin$ $E(G)$, there are edges $v a_{3}, v a_{k-1} \in E(G)$. The edges $v a_{3}, v a_{k-1}$ are not covered by $F$, because otherwise $v \in C_{\text {chord }}$ and thus $\left\{a_{1}, v\right\} \subseteq C_{\text {chord }} \cap C\left(a_{1}, a_{2}\right)$ contradicting the definition of $H$. Hence $v a_{3}, v a_{k-1} \in E(H)$.

$a_{3} \in B_{2}\left[a_{1}\right]$, therefore by the definition of local fragment all edges of $H$ adjacent to $a_{3}$ are covered by $F_{2}\left(a_{1}\right)$. It implies that $F_{2}\left(a_{1}\right) \ni C\left(a_{3}, a_{2}\right), C\left(a_{3}, a_{4}\right)$. Since $v \in C\left(a_{1}, a_{2}\right)$ we have $v \in C\left(a_{3}, a_{4}\right)$. So, $v a_{4} \in E(H)$ (see Figure 1). Note that since $k \geq 7$, we have $a_{4} a_{k-1} \notin E(H)$.

Recall that the vertex $v$ in the local fragment $F_{2}^{\prime}\left(a_{1}\right)$ is covered by the cluster $C^{\prime}\left(v, a_{1}, a_{k}\right)$. All other edges of $H$ incident to $v$ should be covered by at most two clusters of $F_{2}^{\prime}\left(a_{1}\right)$. But this is impossible since the vertices $a_{2}, a_{4}, a_{k-1}$ are pairwise nonadjacent. This contradiction proves Lemma 5 . 


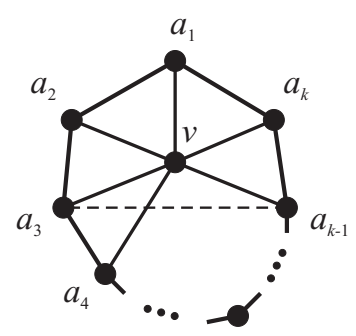

Figure 1:

The considerations above suggest the following algorithm which reduces the problem of recognition chordal graphs with Krausz dimension at most 3 to the same problem for graphs with bounded maximum degree and maximum induced cycle length.

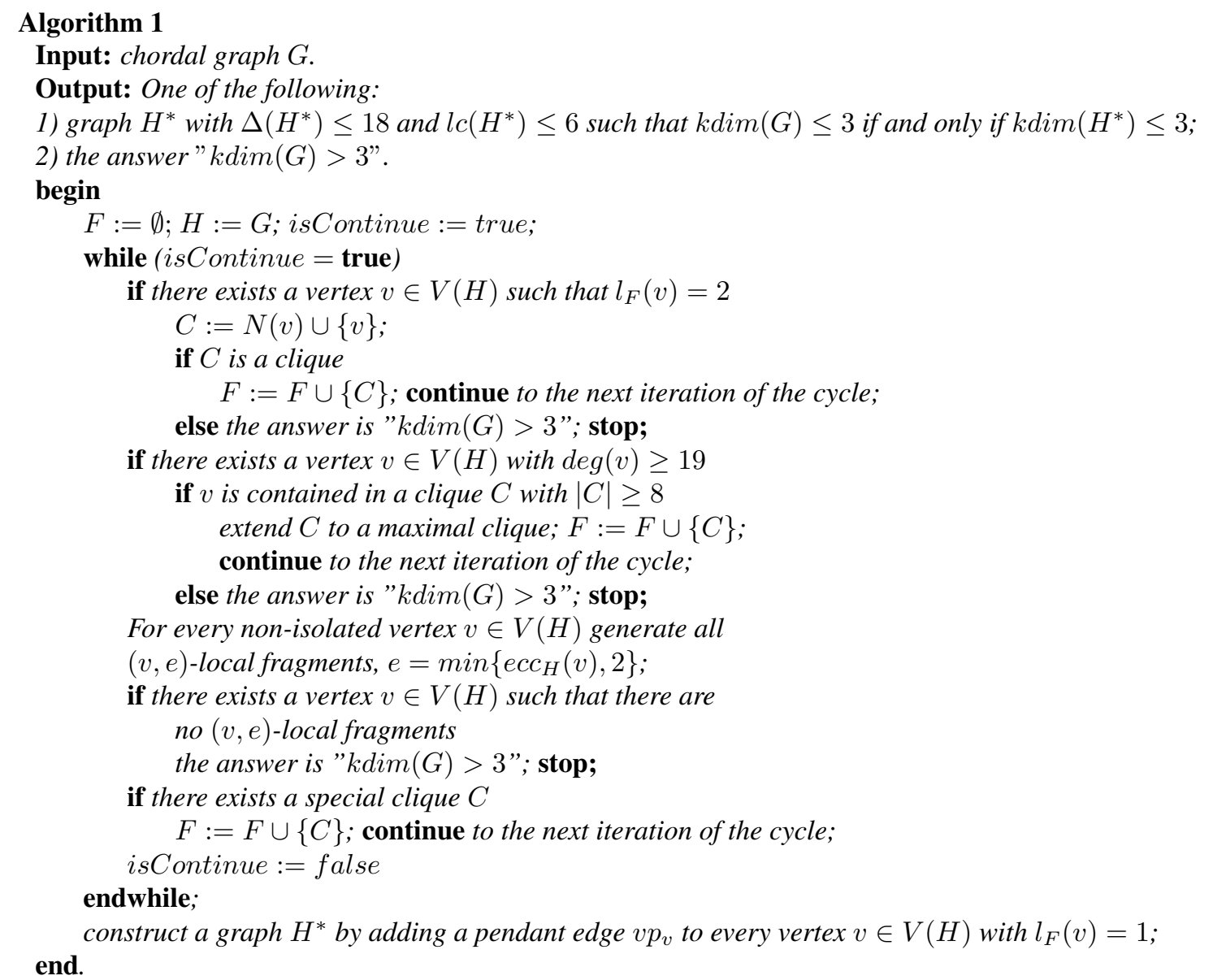


Theorem $6\left([\overline{4]})\right.$ Let $l c\left(H^{*}\right) \leq s+2, \Delta\left(H^{*}\right) \leq \Delta$. Then treewidth $\left(H^{*}\right) \leq \Delta(\Delta-1)^{s-1}$.

Theorem 7 The problem KDIM(3) is polynomially solvable for chordal graph.

Proof: Let us first prove the correctness of Algorithm 1. Once the "while" loop of Algorithm 1 is completed, we obtain the graph $H$ and the set of cliques $F$. By the construction $F$ contains only special cliques. Therefore if the Krausz 3-partition of $G$ exists then it has the form $F \cup R$, where $R$ is a clique partition of $H$ such that every vertex $v \in V(H)$ belongs to at most $3-l_{F}(v)$ clusters of $R$ and every edge of $H$ belongs to exactly one cluster of $R$. By the construction of Algorithm $1 l_{F}(v) \in\{0,1\}$ for every non-isolated vertex $v \in V(H)$. Therefore $R$ is a clique partition of $H$ with required properties if and only if $R \cup\left\{v p_{v}: l_{F}(v)=1\right\}$ is a Krausz 3-partition of $H^{*}$.

Now we will show that the Algorithm 1 is polynomial. Indeed, the procedure of finding large clique which contains the fixed vertex $v \in V(H)$ has the complexity $O(m)$. We start to generate all possible $(v, e)$-local fragments for a vertex $v \in V(H)$ only when $\operatorname{deg}(v) \leq 18$. This implies $\left|B_{e}[v]\right| \leq$ const and thus the complexity of this procedure is constant. The outer loop of Algorithm 1 is performed at most $m$ times.

After performing the Algorithm 1, we obtain the graph $H^{*}$ with bounded maximum degree and the length of the longest induced cycle. By Theorem $6 H^{*}$ has bounded treewidth. For such a graph the problem of determining its Krausz dimension is polynomially solvable [9].

\section{Krausz dimension of split graphs and complements of bipartite graphs}

Theorem 8 The problem KDIM is NP-hard in the class of split graphs.

Proof: We will reduce the problem $K D I M$ for general graphs to the problem $K D I M$ for split graphs.

Let $G$ be a connected graph with $n$ vertices and $m$ edges and $k \leq n-2$ be an integer. Modify $G$ by adding additional vertices and edges as follows:

1. For every $u, v \in V(G)$ such that $u v \notin E(G), u \neq v$ add vertices $x_{u v}, y_{u v}$ and edges $u v, x_{u v} u$, $x_{u v} v, y_{u v} u, y_{u v} v, x_{u v} y_{u v}$. Let $X=\left\{x_{u v}: u v \notin E(G), u \neq v\right\},|X|=\left(\begin{array}{c}n \\ 2\end{array}\right)-m$.

2. For every $u, v, w \in V(G)$ such that $u v \notin E(G)$ and every $w \in V(G) \backslash\{u, v\}$ add a vertex $y_{u v w}$ and edges $x_{u v} w, x_{u v} y_{u v w}, w y_{u v w}$;

3. For every two vertices $x_{u v}, x_{s w} \in X$ add a vertex $z_{u v s w}$ and edges $x_{u v} z_{u v s w}, x_{s w} z_{u v s w}, x_{u v} x_{s w}$;

4. For every $v \in V(G)$ add $n-2-k$ vertices $r_{v, 1}, \ldots, r_{v, n-2-k}$ and edges $v r_{v, 1}, \ldots, v r_{v, n-2-k}$.

Let $H$ be obtained graph (see an example of graph $H$ for the graph $G=\overline{K_{1}+P_{3}}$ on Figure 2). Note, that $A=V(G) \cup X$ and $B=V(H) \backslash A$ is a clique and a stable set in $H$, respectively. So, $H$ is a split graph. We will show, that $G$ has Krausz $k$-partition if and only if $H$ has Krausz $k^{\prime}$-partition, where $k^{\prime}=\left(\begin{array}{l}n \\ 2\end{array}\right)+n-m-2$.

Suppose that $Q$ is Krausz $k^{\prime}$-partition of $H$. For every $u, v \in V(G), u v \notin E(G)$ consider a set

$$
N_{u v}=N\left(x_{u v}\right) \cap B=\left\{y_{u v}\right\} \cup\left\{y_{u v w}: w \in V(G) \backslash\{u, v\}\right\} \cup\left\{z_{u v s w}: x_{s w} \in X \backslash\left\{x_{u v}\right\}\right\}
$$




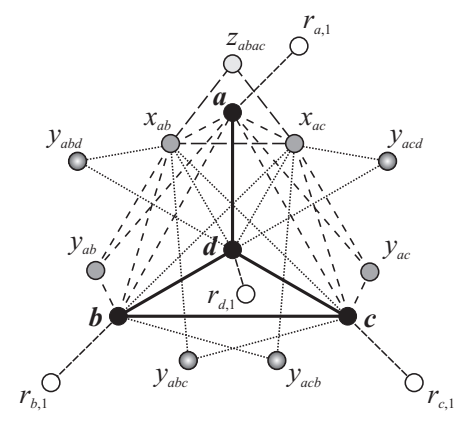

Figure 2:

Let $Q_{u v} \subseteq Q$ be the set of clusters containing $x_{u v} . N_{u v}$ is a stable set, and $\left|N_{u v}\right|=1+(n-2)+\left(\left(\begin{array}{l}n \\ 2\end{array}\right)-\right.$ $m-1)=k^{\prime}$. Therefore every cluster of $Q_{u v}$ contains exactly one vertex from $N_{u v}$, i.e.

$$
Q_{u v}=\left\{C\left(y_{u v}, x_{u v}\right)\right\} \cup\left\{C\left(y_{u v w}, x_{u v}\right): w \in V(G) \backslash\{u, v\}\right\} \cup\left\{C\left(z_{u v s w}, x_{u v}\right): x_{s w} \in X \backslash\left\{x_{u v}\right\}\right\}
$$

Every vertex of $H$, which is adjacent to $x_{u v}$, belongs to one of those clusters. For every vertex from $N\left(x_{u v}\right) \backslash N_{u v}=A \backslash\left\{x_{u v}\right\}$ we have the following.

1. The only vertex from $N_{u v}$, which is adjacent to $u$ and $v$, is $y_{u v}$. Moreover, $N\left(y_{u v}\right)=\left\{u, v, x_{u v}\right\}$. Therefore $C\left(y_{u v}, x_{u v}\right)=\left\{u, v, x_{u v}, y_{u v}\right\}$.

2. The only vertex from $N_{u v}$, which is adjacent to $w \in V(G) \backslash\{u, v\}$, is $y_{u v w}$. Moreover, $N\left(y_{u v w}\right)=$ $\left\{w, x_{u v}\right\}$. Therefore $C\left(y_{u v w}, x_{u v}\right)=\left\{w, x_{u v}, y_{u v w}\right\}$.

3. The only vertex from $N_{u v}$, which is adjacent to $x_{s w} \in X \backslash\left\{x_{u v}\right\}$, is $z_{u v s w}$. Moreover, $N\left(z_{u v s w}\right)=$ $\left\{x_{u v}, x_{s w}\right\}$. Therefore $C\left(z_{u v s w}, x_{u v}\right)=\left\{x_{u v}, x_{s w}, z_{u v s w}\right\}$.

For every $v \in V(G)$ Krausz $k^{\prime}$-partition $Q$ contains clusters $\left\{v, r_{v, i}\right\}, i=1, \ldots, n-2-k$. So, $Q$ contains a fragment

$$
Q^{\prime}=\left\{Q_{u v}: x_{u v} \in X\right\} \cup\left\{\left\{v, r_{v, i}\right\}: v \in V(G), i=1, \ldots, n-2-k\right\} .
$$

For every $v \in V(G)$ let $Q^{\prime}(v)$ be the set of clusters of $Q^{\prime}$ which contain $v$. We have

$$
\begin{aligned}
Q^{\prime}(v)= & \left\{\left\{v, r_{v, i}\right\}: i=1, \ldots, n-2-k\right\} \cup\left\{\left\{u, v, x_{u v}, y_{u v}\right\}: u \in V(G), u v \notin E(G)\right\} \cup \\
& \left.\cup\left\{\left\{x_{s w}, y_{s w v}, v\right\}: s, w \in V(G) \backslash\{v\}, s w \notin E(G)\right\}\right\}
\end{aligned}
$$

So,

$$
\left|Q^{\prime}(v)\right|=n-2-k+\left(\left(\begin{array}{l}
n \\
2
\end{array}\right)-m\right)=k^{\prime}-k
$$

The graph obtained from $H$ by deleting edges covered by $Q^{\prime}$ and isolated vertices is exactly the graph $G$. Consider the fragment $Q^{\prime \prime}=Q \backslash Q^{\prime}$. By (7) $Q^{\prime \prime}$ is Krausz $k$-covering of $G$.

Conversely, it is easy to see, that if $Q^{\prime \prime}$ is Krausz $k$-covering of $G$, then $Q^{\prime} \cup Q^{\prime \prime}$ is Krausz $k^{\prime}$-covering of $H$. 
Corollary 9 The problem KDIM is NP-hard in the class of complements of bipartite graphs.

Proof: Let $G$ be a split graph on $n$ vertices, $V(G)=A \cup B$ be the partition of its vertex set into clique $A$ and stable set $B$ and $k$ be the natural number. Build the graph $H$ as follows:

1. For every $a \in A$ add the new vertex $x_{a}$ and the edge $a x_{a}$;

2. If $n<(k+1)^{2}-(k+1)+2$, add new vertices $y_{1}, \ldots, y_{(k+1)^{2}-(k+1)+2-n}$; let

$$
B^{\prime}=B \cup\left\{x_{a}: a \in A\right\} \cup\left\{y_{1}, \ldots, y_{(k+1)^{2}-(k+1)+2-n}\right\} ;
$$

3. Add all possible edges between vertices of $B^{\prime}$.

$H$ is a complement of bipartite graph. Taking into account Lemma2 it is easy to see that $Q$ is a Krausz $k$-partition of $G$ if and only if $Q \cup\left\{B^{\prime}\right\} \cup\left\{\left\{a, x_{a}\right\}: a \in A\right\}$ is a Krausz $(k+1)$-partition of $H$.

\section{4 m-Krausz dimension of graphs}

We will start with proving the NP-hardness of the problem $K D I M_{m}$.

Theorem 10 The problem $K D I M_{m}$ is NP-hard for every fixed $m \geq 1$.

Proof: Let us reduce to the problem $K D I M_{m}$ the following special case of the 3-dimensional matching problem, which we will call Problem A:

Given: Non-intersecting sets $X, Y, Z$, such that $|X|=|Y|=|Z|=q ; M \subseteq X \times Y \times Z$, such that the following condition holds:

$$
\text { if }(a, b, w),(a, x, c),(y, b, c) \in M \text {, then }(a, b, c) \in M \text {. }
$$

The question: Does $M$ contain a subset $M^{\prime} \subseteq M$ such that $\left|M^{\prime}\right|=q$ and every two elements of $M^{\prime}$ does not have common coordinates? The subset $M^{\prime}$ is called 3-dimensional matching.

It is known that Problem A is NP-complete [8]. Let $X, Y, Z, M,|X|=|Y|=|Z|=q$, be the input of the problem A. Let us reduce problem A to the problem of determining if $k \operatorname{dim}_{m}(G) \leq 2 q-1$. Construct the graph $G$ as follows:

$$
\begin{aligned}
& V(G)=\quad X \cup Y \cup Z \cup\left\{v, v_{1}, \ldots, v_{q-1}\right\} ; \\
& E(G)=\bigcup_{(a, b, c) \in M}\{a b, b c, a c\} \cup\left\{v_{i} v: i=1, \ldots, q-1\right\} \cup\{v d: d \in X \cup Y \cup Z\}
\end{aligned}
$$

(see Figure 3). Let us show that $M$ contains the 3-dimensional matching $M^{\prime}$ if and only if there exists a Krausz $(2 q-1, m)$-partition of $G$.

Suppose that $M^{\prime}=\left\{\left(a_{i}, b_{i}, c_{i}\right): i=1, \ldots, q\right\}$ is the 3-dimensional matching. Let $Q_{1}=\left\{\left\{v, a_{i}, b_{i}, c_{i}\right\}\right.$ : $i=1, \ldots, q\}, Q_{2}=\left\{\left\{v, v_{i}\right\}: i=1, \ldots, q-1\right\}, H=G-E\left(Q_{1} \cup Q_{2}\right)$. All edges adjacent to $v$ are covered by $2 q-1$ cliques of $Q_{1} \cup Q_{2}$ and every vertex $u \in V(G) \backslash\{v\}$ is adjacent to at most $2 q-2$ vertices in $H$. Therefore if $Q_{3}=\{\{z, t\}: z t \in E(H)\}$, then $Q=Q_{1} \cup Q_{2} \cup Q_{3}$ is $\operatorname{Krausz}(2 q-1, m)$-partition of $G$. 


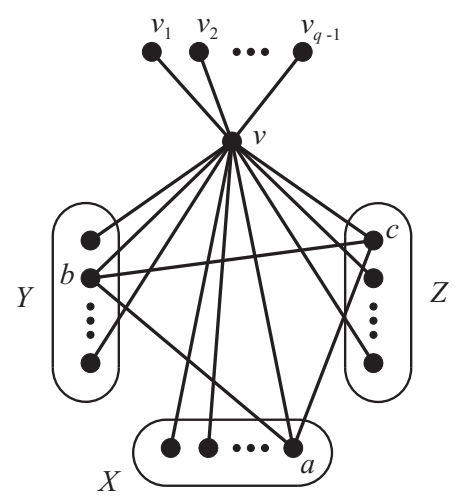

Figure 3:

Let now $Q$ be Krausz $(2 q-1, m)$-partition of $G$. Denote by $Q(v)$ the set of clusters of $Q$, which contain the vertex $v$. Since the vertices $v_{i}, i=1, \ldots, q-1$, have degree 1 , there exist $q-1$ clusters from $Q(v)$ of the form $\left\{v, v_{i}\right\}, i=1, \ldots, q-1$. Let $C_{1}, \ldots, C_{p}$ be the remaining clusters from $Q(v), p \leq q$. Then $\left(C_{1} \cup \ldots \cup C_{p}\right) \backslash\{v\}=X \cup Y \cup Z$. Since $X, Y, Z$ are stable sets of $G$, we have $\left|C_{i}\right| \leq 4, i=1, \ldots, p$. As $|X \cup Y \cup Z|=3 q$, we have $p=q,\left|C_{i}\right|=4, C_{i} \cap C_{j}=\{v\}, i, j=1, \ldots, p, i \neq j$.

Let $C_{i}=\left\{a_{i}, b_{i}, c_{i}, v: a_{i} \in X, b_{i} \in Y, c_{i} \in Z\right\}, i=1, \ldots, q$. The property (*) implies that $M^{\prime}=\left\{\left(a_{i}, b_{i}, c_{i}\right): i=1, \ldots, q\right\} \subseteq M$ and, by the consideration above, $M^{\prime}$ is the 3-dimensional matching.

Now we turn to the complexity of the recognition problem $K D I M_{m}(k)$ in the class of $(\infty, 1)$-polar graphs.

A maximal clique with at least $f(k, m)=m\left(k^{2}-k+1\right)+1$ vertices is called a $(k, m)$-large clique.

In [14] the following two statements were proved. Since they were published only in Russian in a journal that is of difficult access for general readers, we repeat their proofs here.

Theorem 11 Any $(k, m)$-large clique $C$ of a graph $G$ belongs to every Krausz $(k, m)$-partition of $G$.

Proof: Let $A$ be a Krausz $(k, m)$-partition of graph $G, A_{1}, A_{2}, \ldots, A_{t}$ be those clusters of $A$ which have common vertices with $C$. Assume that $C \notin A$. Then the family $B=\left(B_{1}, B_{2}, \ldots, B_{t}\right)$, where $B_{i}=A_{i} \cap C$, is a Krausz $(k, m)$-partition of the graph $G(C)$, and by maximality of $C B_{i} \neq C$ for every $i=1,2, \ldots, t$.

Let us show that $\left|B_{i}\right| \leq m k$ for any $i=1,2, \ldots, t$. Consider a cluster of $B$, say $B_{1}$, and a vertex $u \in C \backslash B_{1}$. No edge of the form $u x$, where $x \in B_{1}$, is contained in $B_{1}$. Moreover, by the definition of Krausz $(k, m)$-partition each cluster of $B$ different from $B_{1}$ contains at most $m$ such edges. Taking into account that the vertex $u$ belongs to at most $k$ clusters of $B$, we obtain the inequality $\left|B_{1}\right| \leq m k$.

Now we will prove that if $B_{i} \backslash B_{j} \neq \emptyset$ for some clusters $B_{j} \in B$, then $\left|B_{j} \backslash B_{i}\right| \leq m(k-1)$. Consider a vertex $u \in B_{i} \backslash B_{j}$. Any edge of the form $u x$, where $x \in B_{j} \backslash B_{i}$ (if such one exists) is contained neither in $B_{i}$, nor in $B_{j}$. Besides, no cluster of $B$ contains more than $m$ of such edges by definition of Krausz $(k, m)$-partition. Taking into account that $u$ belongs to at most $k-1$ clusters of $B$ different from $B_{i}$, we obtain the inequality $\left|B_{j} \backslash B_{i}\right| \leq m(k-1)$. 
Consider an arbitrary vertex $v$ of the clique $C$. Let, without loss of generality, it belong to the clusters $B_{1}, B_{2}, \ldots, B_{s}$ of $B, s \leq t$. We show that $\left|B_{1} \cup B_{2} \cup \ldots \cup B_{s}\right| \leq m k+(s-1) m(k-1)$. The following equality is obvious

$$
\left|B_{1} \cup B_{2} \cup \ldots \cup B_{s}\right|=\left|B_{1}\right|+\left|B_{2} \backslash B_{1}\right|+\left|B_{3} \backslash\left(B_{1} \cup B_{2}\right)\right|+\ldots+\left|B_{s} \backslash\left(B_{1} \cup B_{2} \ldots \cup B_{s-1}\right)\right| .
$$

If $B_{1} \backslash B_{2} \neq \emptyset,\left(B_{1} \cup B_{2}\right) \backslash B_{3} \neq \emptyset, \ldots,\left(B_{1} \cup B_{2} \cup \ldots \cup B_{s-1}\right) \backslash B_{s} \neq \emptyset$, then by the statement proved above, each term in the right part of the equality $(10)$, starting from the second, does not exceed $m(k-1)$. Hence we have $\left|B_{1} \cup B_{2} \cup \ldots \cup B_{s}\right| \leq m k+(s-1) m(k-1)$. Let, on the contrary, $i \in\{2, \ldots, s\}$ be the maximal number such that $\left(B_{1} \cup \ldots \cup B_{i-1}\right) \backslash B_{i}=\emptyset$. Then $B_{1} \subseteq B_{i}, B_{2} \subseteq B_{i}, \ldots, B_{i-1} \subseteq B_{i}$, and the sum of the first $i$ terms in the right part of $(10)$ is equal to $\left|B_{1} \cup B_{2} \cup \ldots \cup B_{i}\right|=\left|B_{i}\right| \leq m k$. Each of the other terms does not exceed $m(k-1)$ by the maximality of $i$. Hence

$$
\left|B_{1} \cup B_{2} \cup \ldots \cup B_{s}\right| \leq m k+(s-i) m(k-1)<m k+(s-1) m(k-1) .
$$

So, in any case we obtain that the inequality $\left|B_{1} \cup B_{2} \cup \ldots \cup B_{s}\right| \leq m k+(s-1) m(k-1)$ holds. Taking into account that $C=B_{1} \cup B_{2} \cup \ldots \cup B_{s}$ and $s \leq k$, we have

$$
|C| \leq m k+(k-1) m(k-1)=m\left(k^{2}-k+1\right)<f(k, m) .
$$

The obtained contradiction proves the lemma.

Theorem 12 For every fixed $k, m \geq 1$, there exists a finite set $\mathcal{F}_{0}$ of forbidden induced subgraphs such that a split graph $G$ belongs to the class $L_{k}^{m}$ if and only if no induced subgraph of $G$ is isomorphic to an element of $\mathcal{F}_{0}$.

Proof: Denote by $R_{p}$ the graph obtained from the complete graph $H \cong K_{f(k, m)}$ by adding a new vertex and connecting it with exactly $p$ vertices of $H$. Put $\mathcal{F}_{0}=\left\{R_{p}: k m+1 \leq p \leq f(k, m)-1\right\} \cup\left\{K_{1, k+1}\right\}$. Using Theorem 11 one can immediately verify that no graph from $\mathcal{F}_{0}$ belongs to $L_{k}^{m}$.

Let, without loss of generality, $G$ be a connected graph, and $V(G)=C \cup S$ be a bipartition of $V(G)$ into clique $C$ and stable set $S$ such that $C$ is a maximal clique. Let also no induced subgraph of $G$ be isomorphic to an element of $\mathcal{F}_{0}$. Put $S=\left\{v_{1}, \ldots, v_{s}\right\}$. Consider two cases:

1) $|C|>(k m-1) k+1$.

In this case we have

$$
|C| \geq(k m-1) k+2=m k^{2}-(k-1)+1 \geq m k^{2}-m(k-1)+1=f(k, m) .
$$

Then, since no induced subgraph of $G$ is isomorphic to a graph $R_{p}, k m+1 \leq p \leq f(k, m)-1$, we have $\operatorname{deg}\left(v_{i}\right) \leq k m$ for any $i=1,2, \ldots, s$. Since $G$ contains no induced $K_{1, k+1}$, we have $|N(u) \cap S| \leq k$ for any vertex $u$ from $C$. Moreover, we prove that for any vertex $u$ from $C$ the inequality $|N(u) \cap S| \leq k-1$ holds. Assume this is not true. Let, without loss of generality, some vertex $u$ from $C$ be adjacent to the vertices $v_{1}, \ldots, v_{k}$ from $S, k \leq s$. Since $\operatorname{deg}\left(v_{i}\right) \leq k m, i=1,2, \ldots, k$, and $u \in \bigcap_{i=1}^{k} N\left(v_{i}\right)$, then $\left|\bigcup_{i=1}^{k} N\left(v_{i}\right)\right| \leq \sum_{i=1}^{k}\left(\operatorname{deg}\left(v_{i}\right)-1\right)+1 \leq(k m-1) k+1<\varphi(G)$. Hence, there exists a vertex $u^{\prime}$ 
from $C$, which is not adjacent to any vertex from $v_{1}, \ldots, v_{k}$. But then $G\left(u, u^{\prime}, v_{1}, \ldots, v_{k}\right) \cong K_{1, k+1}$, a contradiction.

Now we can construct a Krausz $(k, m)$-partition of $G$. Since $\operatorname{deg}\left(v_{i}\right) \leq k m$ for any $i=1,2, \ldots, s$, then there exists a partition $N\left(v_{i}\right)=C_{i_{1}} \cup \ldots \cup C_{i_{s_{i}}}$, where $C_{i_{j}} \cap C_{i_{l}}=\emptyset, j, l \in\left\{1, \ldots, s_{i}\right\}, j \neq l$, $\left|C_{i_{j}}\right| \leq m, s_{i} \leq k$. Obviously, the list of cliques $\left\{C_{i_{j}} \cup\left\{v_{i}\right\}: i=\overline{1, s}, j=\overline{1, s_{i}}\right\}$ together with the clique $C$ is a $\operatorname{Krausz}(k, m)$-partition of graph $G$.

2) $|C| \leq(k m-1) k+1$.

Since $G$ contains no induced $K_{1, k+1}$, we have $|N(u) \cap S| \leq k$ for any vertex $u$ from $C$. Therefore, as $G$ is connected,

$|G|=|C|+|S| \leq|C|+\sum_{u \in C}|N(u) \cap S| \leq((k m-1) k+1)+((k m-1) k+1) k=((k m-1) k+1)(k+1)$,

i. e., the order of graph $G$ is bounded above by a value, depending on $k$ and $m$. Add to the list $\mathcal{F}_{0}$ all such split graphs $H$, that $H \notin L_{k}^{m}$ and $|H| \leq((k m-1) k+1)(k+1)$.

Obviously, the constructed in the cases 1) and 2) finite list $\mathcal{F}_{0}$ is a required list of forbidden induced subgraphs.

Since $K_{1, k+1} \notin L_{k}^{m}$, the heredity of $L_{k}^{m}$ immediately implies

Lemma 13 A bipartite graph $G$ belongs to the class $L_{k}^{m}$ if and only if no induced subgraph of $G$ is isomorphic to $K_{1, k+1}$.

Theorem 14 For every fixed $k, m \geq 1$, there exists a finite set $\mathcal{F}$ of forbidden induced subgraphs such that an $(\infty, 1)$-polar graph $G$ belongs to the class $L_{k}^{m}$ if and only if no induced subgraph of $G$ is isomorphic to an element of $\mathcal{F}$.

Proof: Without loss of generality, we can suppose that $(\infty, 1)$-polar graph $G$ is connected. Let $G$ have bipartition $(A, B) ; A_{i}, i=1,2, \ldots, t$, be the vertex sets of connected components of $\bar{G}(A) ; \mathcal{F}_{0}$ be the set of split graphs from Theorem 12 Denote by $\mathcal{F}_{1}$ the set of $(\infty, 1)$-polar graphs which do not belong to the class $L_{k}^{m}$ and have order at most $(k+1) k(f(k, m)-1)$.

Put $\mathcal{F}=\mathcal{F}_{0} \cup \mathcal{F}_{1} \cup\left\{K_{1, k+1}, K_{f(k, m)+1}-e\right\}$, where $K_{f(k, m)+1}-e$ is the graph obtained from the complete graph $K_{f(k, m)+1}$ after deleting an edge. The set $\mathcal{F}$ is finite, since $\mathcal{F}_{0}$ and $\mathcal{F}_{1}$ are finite. According to Theorem 11 , there is no $\operatorname{Krausz}(k, m)$-partition for $K_{f(k, m)+1}-e$. Therefore, $K_{f(k, m)+1}-$ $e \notin L_{k}^{m}$. Thus, $\mathcal{F} \cap L_{k}^{m}=\emptyset$. The necessity of the statement follows from the heredity of the class $L_{k}^{m}$.

Now let $G$ contain no induced subgraph isomorphic to an element from $\mathcal{F}$. If $G(A)$ is complete, then $G$ is a split graph and by Theorem $12 G \in L_{k}^{m}$. If $G(A)$ is empty, then $G$ is a bipartite graph and by Lemma $13 G \in L_{k}^{m}$.

Now suppose that $G(A)$ is neither complete nor empty graph. Then $2 \leq t \leq|A|-1$. Since $K_{1, k+1} \in$ $\mathcal{F}$, then $\left|A_{i}\right| \leq k$ for any $i=1,2, \ldots, t$. Now we will prove that since $K_{f(k, m)+1}-e \in \mathcal{F}$, then $t \leq f(k, m)-1$. Let, to the contrary, $t \geq f(k, m)$. As $G(A)$ is not a complete graph, there exists an index $i_{0} \in\{1,2, \ldots, t\}$ such that $\left|A_{i_{0}}\right| \geq 2$. Consider the set $S=\left\{a_{1}, a_{2}, \ldots, a_{i_{0}-1}, a_{i_{0}}^{\prime}, a_{i_{0}}^{\prime \prime}, a_{i_{0}+1}, \ldots, a_{t}\right\}$, where $a_{i} \in A_{i}$ for any $i \in\{1,2, \ldots, t\} \backslash\left\{i_{0}\right\}$ and $a_{i_{0}}^{\prime}, a_{i_{0}}^{\prime \prime} \in A_{i_{0}}$. Then $G(S)$ contains $K_{f(k, m)+1}-e$ as an induced subgraph, a contradiction. Therefore

$$
|A| \leq \sum_{i=1}^{t}\left|A_{i}\right| \leq k(f(k, m)-1) .
$$


Since $|N(a) \cap B| \leq k$ for any vertex $a \in A$ and $G$ is connected, we have

$|G| \leq|A|+|B| \leq|A|+\sum_{a \in A}|N(a) \cap B| \leq k(f(k, m)-1)+k^{2}(f(k, m)-1)=(k+1) k(f(k, m)-1)$.

It follows from the inclusion $\mathcal{F}_{1} \subseteq \mathcal{F}$ that $G \in L_{k}^{m}$.

Corollary 15 The problem $K D I M_{m}(k)$ is fixed parameter tractable in the class of $(\infty, 1)$-polar graphs, when parameterized by $k, m \geq 1$.

Proof: The proofs of Theorems 12, 14 and Lemma 13 imply the following algorithm of recognition whether a given $(\infty, 1)$-polar graph belong to the class $L_{k}^{m}$.

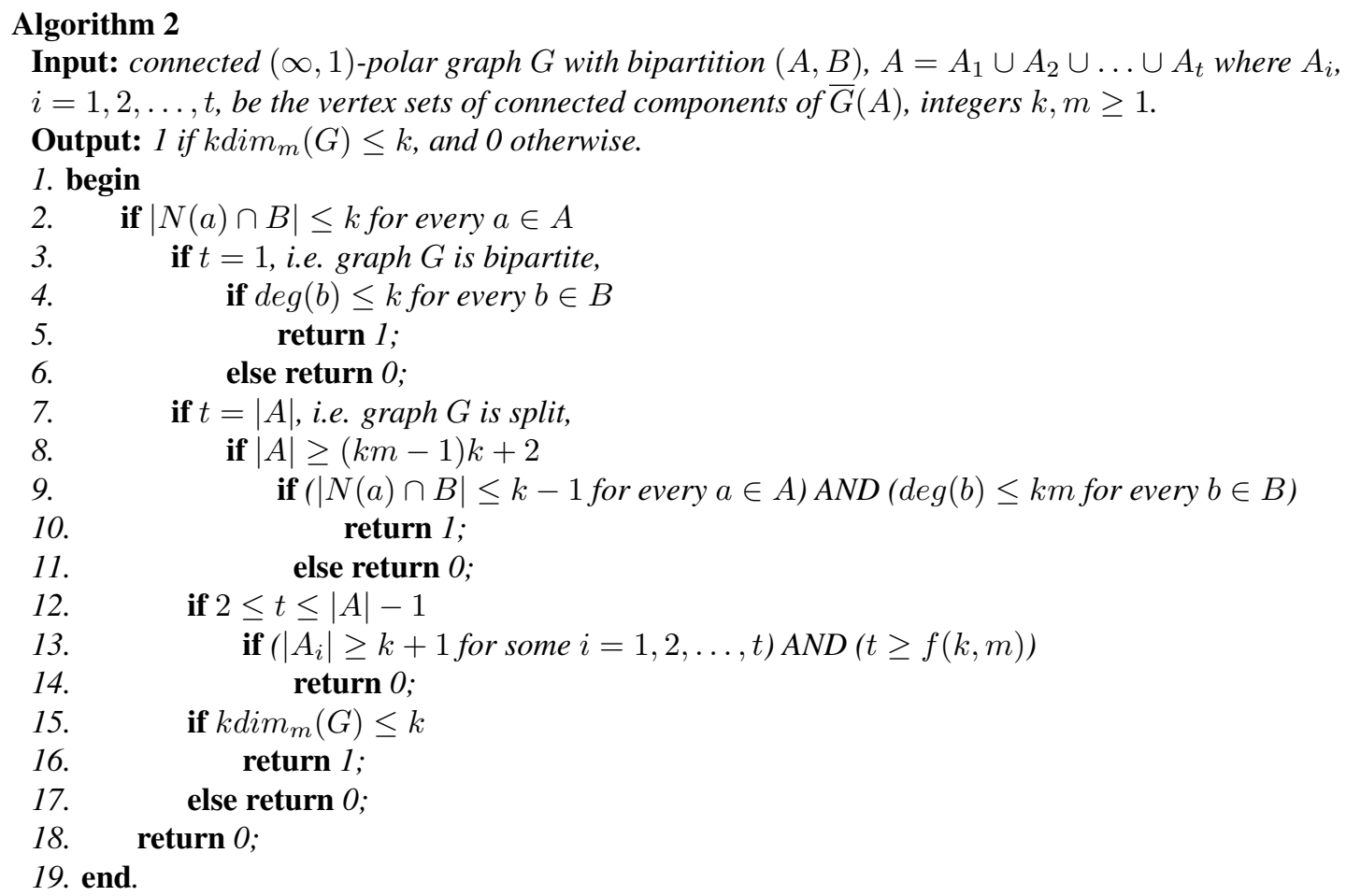

The complexity of the algorithm in lines $1-14$ is $O\left(n^{2}\right)$ at worst, where $n=|V(G)|$. Since the order of the graph $G$ in line 15 is bounded above by value $g(k, m)=\max \{((k m-1) k+1)(k+1),(k+$ $1) k(f(k, m)-1)\}$ the problem " $k \operatorname{dim}_{m}(G) \leq k$ " in this line could be solved in time $O(h(k, m))$, where $h$ is some function.

So, the total complexity of Algorithm 2 is $O\left(n^{2}+h(k, m)\right)$.

\section{Acknowledgements}

This work was partially supported by Belarussian Republican Fund of Fundamental Research. 


\section{References}

[1] Beineke L.W. Derived graphs and digraphs, Beitrage zur Graphentheorie, Leipzig (1968), 17-33.

[2] Beineke L.W., Broere I. The Krausz dimension of a graph, Utilitas Mathematica 69 (2006), 183-194.

[3] Berge C. Hypergraphs. Combinatorics of Finite Sets, North-Holland Mathematical Library, Amsterdam, 1989.

[4] Bodlaender H.L., Thilikos D.M. Treewidth for graphs with small chordality, Discr. Appl. Math. 79 (1997), 45-61.

[5] Zh. Chernyak, A. Chernyak. About recognizing $(\alpha, \beta)$ classes of polar graphs, Discr. Math. 62(2) (1986), 133138.

[6] Degiorgi D.G., Simon K. A dynamic algorithm for line graph recognition, Proc. WG'95, Lecture Notes in Computer Science 1017 (1995), 37-48.

[7] de Werra, D., Ekim, T., Hell, P. and Stacho., J. Polarity of chordal graphs, Discrete Applied Math. 156 (2008) 2469-2479.

[8] Garey M.R., Johnson D.S. Computers and Intractability. A Guide to the Theory of NP-completeness, San Francisco, CA, 1979.

[9] Hliněný P., Kratochvíl J. Computational complexity of the Krausz dimension of graphs, Proc. WG '97, Lecture Notes in Computer Science 1335 (1997), 214-228.

[10] Jacobson M.S., Kezdy A.E., Lehel J. Recognizing intersection graphs of linear uniform hypergraphs, Graphs and Comb. 4 (1997), 359-367.

[11] Levin A.G. and Tyshkevich R.I. Line Hypergraphs, Diskret. Mat. 5 (1993), No. 1, 112-129.

[12] Lehot P.G.H. An optimal algorithm to detect a line graph and output its root graph, J. Assoc. Comput. Mach. 21, 569-575.

[13] Metelsky Yu. Split intersection graphs of hypergraphs with bounded rank, Vestsi Nats. Akad. Navuk Belarusi. Ser. Fiz.-Mat. Navuk (1997) No. 3, 117-122.

[14] Metelsky Yu., Schemelyova K.N. Finite characterizability of intersection graphs of hypergraphs with bounded rank and multiplicity in the class of split graphs, Vestn. Beloruss. Gos. Univ. Ser. 1 Fiz. Mat. Inform. 2008, No. 1, 102-105.

[15] Metelsky Yu., Tyshkevich R. Line graphs of linear 3-uniform hypergraphs, J. Graph Theory 25 (1997), 243251.

[16] Naik R.N., Rao S.B., Shrikhande S.S., Singhi N.M. Intersection graphs of k-uniform linear hypergraphs, Ann. Discrete Math. 6 (1980), 275-279.

[17] Naik R.N., Rao S.B., Shrikhande S.S., Singhi N.M. Intersection graphs of k-uniform linear hypergraphs, Europ. J. Combin. 3 (1982), 159-172.

[18] Naor J., Novick M.B. An efficient reconstruction of a graph from its line graph in parallel, J. Algorithms 11 (1990), 132-143.

[19] Roussopoulos N.D. A $\max \{\mathrm{m}, \mathrm{n}\}$ algorithm for determining the graph H from its line graph G, Inform. Process. Lett. 2 (1973), 108-112.

[20] Skums P.V., Suzdal S.V., Tyshkevich R.I. Edge intersection graphs of linear 3-uniform hypergraphs. Discrete Math. 309 (2009), 3500-3517.

[21] Tyshkevich, R. and Chernyak, A. Unigraphs, Isv. Akad. Nauk BSSR, Ser. Fiz.-Mat. Nauk 5, 1978, 5-11 